\title{
On the physically consistent characterisation of a system for FRF-based substructuring
}

\author{
Nicola Contartese ${ }^{\mathrm{a}, \mathrm{b}, *}$, Eugène Nijman $^{\mathrm{a}}$, Wim Desmet ${ }^{\mathrm{b}, \mathrm{c}}$ \\ ${ }^{a}$ Virtual Vehicle Research Gmbh, Inffeldgasse 21a, 8010 Graz, Austria \\ ${ }^{b} K U$ Leuven, Department of Mechanical Engineering, Celestijnenlaan 300, B-3001 Heverlee, Belgium \\ ${ }^{c}$ DMMS Core Lab, Flanders Make, Belgium
}

\begin{abstract}
Substructuring approaches have been extensively used since long time to predict the vibroacoustic behaviour of built-up mechanical systems. In the frequency domain, these methods build on the dynamic characterisation of the subsystems via frequency response functions at the coupling interfaces. Intensively used for subsystems connected by points, dynamic substructuring still constitutes an open area of research for systems comprising continuous interfaces. Within this framework, a method which allows to characterise subsystems connected along lines is presented. The approach is based on a discretisation of the continuous interfaces into a small set of points, where the information of the subsystems is condensed. For this purpose, an inverse approach is used, which allows to characterise the dynamics of the passive subsystem using data of the assembled system. A straightforward inversion, however, does not assure the physical consistency of the characterized subsystem. In other words, the subsystem extraction procedure must not only guarantee correct dynamic behaviour of the assembled system, but the obtained subsystem must also be reciprocal and passive. The central theme of this paper is the formulation of a constrained optimisation procedure for the enforcement of reciprocity and passivity on the inversely obtained subsystem characteristics. The method is validated on a structure made of two plates connected along a common edge through a beam.
\end{abstract}

Keywords: System characterisation, Physical consistency, Substructure coupling, Interface problem, Inverse method

\section{Introduction}

Over the last decades, dynamic substructuring has represented a valuable engineering tool for the analysis of built-up mechanical systems. The methodology, which finds its roots in the field of domain decomposition, consists of dividing a complex structure into smaller and more manageable components. Their individual analysis and subsequent assembly allow to predict the dynamic behaviour of the total system in an efficient manner. The most widespread techniques employed in dynamic substructuring are based on Component Mode Synthesis (CMS) and Frequency Response Function (FRF) coupling methods. The starting point of a CMS analysis is to identify sets of modal basis functions which approximate the dynamic behaviour of the individual substructures. Then, compatibility and equilibrium conditions are enforced along the interfaces between the components. Introduced by Hurty [1] in 1965, this method has been developed over time with manifold variants 2, 3, 4, differing mainly in the nature of the modes used to build the reduction space. As an alternative to CMS approaches, FRF techniques use directly the frequency responses of the uncoupled components to reproduce the dynamic response of the total system. Hence, errors such as modal truncation, introduced during the modal identification step, are avoided. The classical FRF-based substructuring (FBS) method has been proposed by Jetmundsen et al. [5] in 1988. Also known as admittance modelling, it consists

\footnotetext{
*Corresponding author at: Virtual Vehicle Research Gmbh, Inffeldgasse 21a, 8010 Graz, Austria.

Email address: nicola.contartese@v2c2. at (Nicola Contartese)
} 
in the coupling of the receptance matrices of the substructures. Several studies have been conducted over time towards the improvement of the computational efficiency and robustness of the FBS method. For instance, filtration techniques have been developed by $\mathrm{Lim}$ and $\mathrm{Li}$ [6] to reduce the error amplification during the inversion calculation and by Carne and Dohrmann [7] and Peeters et al. [8] to remove unphysical behaviours of the FRFs. An extended review of FRF based approaches later on is presented by de Klerk at al. in 9 .

\subsection{Reduction of the interface problem size}

Whether a CMS or an FRF-based coupling method is implemented, a cumbersome issue remains the computational efficiency of the interface problem. In many practical cases the number of Degrees of Freedom (DoFs) on the interface is high and the traditional substructuring methods become prohibitively inefficient. This situation turns to be even more critical when two substructures are connected along a continuous interface as happens in many industrial applications, e.g. when internal stiffening frames are connected along lines or when parts are seamwelded together. Apart from the purely numerical settings, the situation where the structural assembly contains experimentally characterised component systems is particularly challenging. A method used to reconstruct the interface dynamics setting out from a discrete number of measured points is the system equivalent reduction expansion process (SEREP) [10], which uses static deformations obtained from a finite element model to create a mapping between measured and unmeasured DoFs. A different approach to the problem was successively proposed by Bonhoff and Petersson [11. The characterisation of the interface mobility is addressed by expanding the force and velocity fields into Fourier series, which are subsequently used for transmission problems that involve circular interfaces located on plate-like structures. Another methodology was then proposed by Donders et al. [12. With his wave-based substructuring (WBS) approach, the interface representation between substructures is reduced by expressing the interface DoFs in terms of a limited set of basis functions, so called waves. Later on, Meyer at al. 13 proposed the Condensed Transfer Function (CTF) method. In this case, a set of orthonormal functions, or condensation functions, is defined along the coupling line and used as a basis for approximating the displacement and force fields along the junction. In the study presented in this paper, the interface reduction problem is addressed by discretising a continuous connection into few points.

\subsection{Substructuring using inverse methods}

This work addresses the possibility to approximate continuously coupled systems in a broad frequency range by point coupled systems using an inverse approach. The scope of this work is to achieve this while enforcing physical consistency, more in particular reciprocity and passivity, upon the inversely obtained subsystems. Also known as decoupling problem or subsystem subtraction, inverse dynamic substructuring aims to identify the dynamic behaviour of a substructure starting from the known dynamic behaviour of the coupled system. This technique becomes particularly relevant when the individual characterisation of the subsystems is cumbersome such as for substructures that are very delicate or in operational conditions [14. Over the last two decades, a large body of studies has been published with regard to inverse substructure characterisation. These include impedance and mobility based approaches [15] or methods based on the dual assembly [16. Some examples are the works of Ind and Ewins [14] and D'Ambrogio and Fregolent [17, formulated in the modal domain. In order to avoid errors introduced by the modal truncation the same authors later also published FRF-based decoupling procedures [18, 19. Moorhouse et al. [20] proposed a method for the indirect determination of the mobility of structure-borne sound sources, i.e. structures with an internal excitation. Instead of performing measurements on the subsystems in the free state, the source mobility is obtained from measurements on the coupled structure and on the decoupled receiver substructure. The method has the practical advantage that no blocked force test rig is needed. Nevertheless, the twostage measurement procedure requires force excitations both on the stand-alone source and on the coupled receiver substructure. Holler and Gibbs 21 further developed this method and introduced a formulation which requires a force excitation applied on the receiver structure only, not on the source itself. Even though no measurements at the contact points in the coupled state is needed, multiple mobility terms of the coupled structure and the receiver substructure have to be evaluated. An inverse substructuring method that does 
not require component-level FRFs was instead proposed by Zhen et al. 22, which allows to characterise the unknown substructure purely from system-level FRFs. Wang et al. [23, 24] further extended this method to multi-substructure coupled systems by introducing a step-by-step decoupling approach. Furthermore, parallel works 25] have recently extended the inverse sub-structuring method for the decoupling of nonlinear systems when the nonlinear effects cannot be neglected. However, in most of the above mentioned studies little or no attention was paid to the fact that the inverse problem might lead to physically inconsistent subsystem models, i.e. models that violate the first principles of linear structural dynamics. This specific point was addressed partially by Peeters et al. [8] and Carne and Dohrmann [7] and more in depth by Sjövall and Abrahamson [26. In the latter study a subsystem is characterised setting out from a physically consistent model of the coupled system in a state-space representation. The enforcement of physical consistency of the inversely obtained subsystems will also be the central theme of the current paper.

\subsection{Scope and structure}

This study presents an inverse method that allows not only to characterise the dynamic behaviour of a receiver subsystem connected along a continuous interface but also to ensure its physical consistency. While an infinite number of DoFs would be required to account for their interaction, this approach considers only a few points at the connection making use of an inverse approach. The purpose of this paper is to not only mimic the continuously coupled system response as accurately as possible but also to do so safeguarding the physically consistent behaviour of the point connected system. As compared to the conventional procedure, the benefits provided by the presented technique are twofold: (i) it requires measurements only in a condensed set of points at the interface and (ii) it gives as output an impedance model which is sure to be physically consistent. A concise overview of the problem tackled in this study is presented in Section 2, The dynamic behaviour of a receiver subsystem connected along a continuous interface is characterised by using few interface points. For this scope, the inverse problem described in Section 3 is needed. The discrete source mobility and free velocities, both evaluated at the coupling points, are known. The interface velocities of the continuously coupled system are also given as input. Then, the discrete receiver impedance is evaluated at the coupling points. Particular attention is paid that the identified receiver subsystem does not violate first principle properties of linear structural dynamic systems. Section 4 focuses on the reciprocity and passivity properties, which are subsequently enforced during the subsystem characterisation described in Section 5 . A concise overview of this approach can be found in Figure 2 of Section 5.4. In Section 6, the accuracy of the method is demonstrated on the basis of an application case. A system made of two plates connected together through a beam is analysed. Finally, Section 7 draws the conclusions.

\section{Problem definition}

Consider a dynamic system as schematically represented in Figure 1. Two subsystems can be distinguished: an active (source) subsystem 1 containing an excitation $\mathbf{f}_{S}(\omega)$ and a passive (receiver) subsystem 2. It is assumed that the subsystems are linear, time-invariant (LTI) and rigidly interconnected along a line junction. Any relative motion between the two subsystems is therefore not allowed. This continuous interface is discretised into a limited number of points hereinafter called coupling points, so that a set of $n$ coupling DoFs are taken into account. We are interested in the velocity response spectra $\mathbf{v}_{R 1}(\omega)$ and $\mathbf{v}_{R 2}(\omega)$ at the receiving locations due to the force excitation $\mathbf{f}_{S}(\omega)$.

The first step of a classical FRF based substructure method consists in determining the dynamic characteristics of active and passive subsystems by means of interface transfer functions. The subsequent coupling between both subsystems allows to identify the force field at the common interface. Finally, the response is built up from the interface forces and an appropriate set of transfer functions relating them to the receiving response. In what follows, we aim to condense the dynamic characteristics of the passive side in a discrete number of interface DoFs. Hence, an inverse approach has to be performed. 


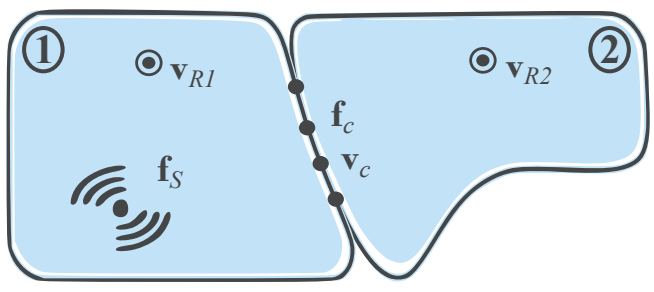

(a) coupled system

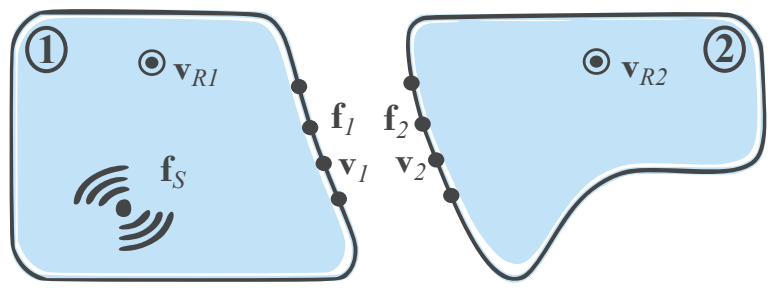

(b) uncoupled subsystems

Figure 1: Schematic representation of a build-up system in the continuously coupled $1 \mathrm{a}$ and uncoupled state 1b.

\section{Inverse subsystem characterisation}

In this framework, the description of a subsystem relies on the more general concept of condensed impedance, as employed by Grialou [27. The internal DoFs that are not directly excited or measured are "removed" or condensed on the interface. As a result, the subsystems are treated in terms of black boxes [28, with their input-output characteristics described without the need for a detailed description of its inner workings. Analogies with the presented method can be also found in the Operational Transfer Path Analysis (OTPA) concept, described for instance in Allen et al. 29].

\subsection{Condensed impedance}

For each uncoupled subsystem $\alpha \in\{1,2\}$ shown in Figure 1, the condensed impedance relates the vector of interface forces $\mathbf{f}_{\alpha}(\omega) \in \mathbb{C}^{n \times 1}$ and interface velocity $\mathbf{v}_{\alpha}(\omega) \in \mathbb{C}^{n \times 1}$ (where $n$ is the number of coupling DoFs) in the form:

$$
\mathbf{f}_{\alpha}(\omega)=\mathbf{Z}_{\alpha}(\omega) \mathbf{v}_{\alpha}(\omega) .
$$

$\mathbf{Z}_{\alpha}(\omega) \in \mathbb{C}^{n \times n}$ describes the forces needed to generate a unit harmonic velocity on one particular interface DoF while keeping all the other interface DoFs fixed. Its ij entry reads:

$$
Z_{\alpha, i j}(\omega)=\frac{f_{\alpha, i}(\omega)}{v_{\alpha, j}(\omega)} \quad v_{\alpha, k \neq j}=0,
$$

where the subscripts $i$ and $j$ denote the elements in the full set of interface DoFs. The concept of condensed impedance represents an inherent dynamic property of the subsystem and consequently remains unchanged regardless of whether the subsystem is coupled or not. If we consider the condensed impedance matrix of the receiver substructure in the coupled state shown in Figure 1a interface velocities and forces are influenced by the presence of the source. We refer in this case to coupled forces $\mathbf{f}_{c}(\omega) \in \mathbb{C}^{n \times 1}$ and coupled velocities $\mathbf{v}_{c}(\omega) \in \mathbb{C}^{n \times 1}$, and Eq. (1) can be rewritten for the receiver subsystem as

$$
\mathbf{f}_{c}=\mathbf{Z}_{2} \mathbf{v}_{c} .
$$

From here on, the explicit frequency dependency is omitted to improve readability. Eq. (3) is valid for any configuration of interface excitations coming from the interaction with the source substructure: for a generic set of input velocities $\mathbf{v}_{c}$ there will be a set of output forces $\mathbf{f}_{c}$ determined by the impedance matrix $\mathbf{Z}_{2}$. Hence, if the source subsystem is solicited with $m$ independent excitation configurations, Eq. (3) can be repeated $m$ times and the corresponding $\mathbf{f}_{c}$ and $\mathbf{v}_{c}$ vectors can be stored as columns in the matrices $\mathbf{F} \in \mathbb{C}^{n \times m}$ and $\mathbf{V} \in \mathbb{C}^{n \times m}$, respectively:

$$
\left[\begin{array}{llll}
\mathbf{f}_{c}^{(1)} & \mathbf{f}_{c}^{(2)} & \cdots & \mathbf{f}_{c}^{(m)}
\end{array}\right]=\mathbf{Z}_{2}\left[\begin{array}{llll}
\mathbf{v}_{c}^{(1)} & \mathbf{v}_{c}^{(2)} & \cdots & \mathbf{v}_{c}^{(m)}
\end{array}\right] \Rightarrow \mathbf{F}=\mathbf{Z}_{2} \mathbf{V}
$$

The superscripts $1,2, \ldots, m$ refer to several excitation configurations, hereinafter called load cases. The impedance matrix $\mathbf{Z}_{2}$ can be evaluated if the number of independent load cases $m$ is larger than or equal to the number of coupling DoFs $n$. An over-determined system of equations is however preferred in view 
of the regularising effect on the transfer function estimate. Thus, the impedance matrix of subsystem 2 is found as

$$
\mathbf{Z}_{2}^{e q}=\mathbf{F V}^{\dagger},
$$

where the superscript $\dagger$ stands for the Moore-Penrose inverse, which is assumed to exist. Eq. (5) represents the inverse problem which allows to characterise a passive subsystem starting from the knowledge of the coupled state. The superscript $e q$ stands for equivalent and highlights the fact that the identified impedance matrix, hereinafter called equivalent impedance matrix, is not equal to the physical one, i.e. the one obtained by directly exciting the interface DoFs of the standalone subsystem. This difference is mainly due to the fact that $\mathbf{Z}_{2}^{e q}$ condenses the dynamic behaviour of the receiver subsystem into few coupling points lying on a continuous interface. Hence, $\mathbf{Z}_{2}^{e q}$ might be considered as the impedance matrix of a fictitious receiver subsystem that, attached to the source through few coupling points, mimics the dynamic influence of the real receiver subsystem along a continuous interface.

\subsection{Evaluation of coupled forces}

The matrix $\mathbf{F}$ of Eq. (4) contains interface forces $\mathbf{f}_{c}$ that cannot be assessed directly and need to be identified by other means. By invoking the superposition principle as described by Bobrovnitskii [30, we can consider the coupled velocity $\mathbf{v}_{c}$ as the sum of two contributions:

$$
\mathbf{v}_{c}=\mathbf{v}_{\text {free }}+\mathbf{Y}_{1} \mathbf{f}_{c}
$$

The first right hand side term $\mathbf{v}_{\text {free }}$ is the so called free velocity, i.e. the interface velocity of the stand-alone source substructure when it is excited by the internal operational forces. The second term is given by the multiplication of the coupled forces with the source mobility matrix $\mathbf{Y}_{1}$. It represents the velocity response due to the interface forces of the coupled system when the internal sources are switched off. At this point, Eq. (6) can be arranged so that the vector of coupled forces is indirectly obtained as:

$$
\mathbf{f}_{c}=\mathbf{Y}_{1}^{-1}\left(\mathbf{v}_{c}-\mathbf{v}_{\text {free }}\right) \text {. }
$$

By adopting this approach, no force measurements at the coupling points are required to create the columns of the matrix $\mathbf{F}$ in Eq. (4). In case of an experimental characterisation, this procedure would however require testing in both a coupled and a free-interface condition, which could in practice involve measurement inconsistencies as highlighted by Meggitt and Moorhouse [31. The outlined procedure is repeated for all frequencies so that the equivalent impedance matrix is computed in the full frequency range of interest.

\section{Physically consistent model}

One of the major concerns when dealing with an inverse problem is the accuracy of the obtained results. However, accuracy is not the only feature for determining the quality of a dynamic model. One of the purposes of this study is to show that first principle properties of LTI structural systems have to be guaranteed. As previously stated in Section $3, \mathbf{Z}_{2}^{e q}$ is computed in a least-squares sense. Hence, a solution which does not fulfil some basic properties of physical passive systems can be obtained. The need to create a physically consistent model on a subsystem level has been extensively posed in the past, since it affects the accuracy of the reconstruction of the dynamic behaviour on a system level. For instance, Carne and Dohrmann [7] showed that small hidden anomalies in the FRF subsystem matrices, coming from both measurement noise and subsequent mathematical processing of the FRFs, can substantially affect the dynamic behaviour of the assembly.

Two important properties that a structural dynamic LTI system must exhibit in order to be physically consistent are reciprocity and passivity. Reciprocity implies that sensors and actuators can be interchanged, rendering a symmetric impedance matrix. Passivity denotes that the total vibrational power supplied to a structure must be positive or, in the ideal case of undamped structures without energy dissipation, null. In other words, a passive system is unable to generate energy. As widely reported in system and control theory, see e.g. Åström and Wittenmark [32, Willems [33] or Triverio et al. 34], the concept of passivity 
is closely related to the property of positive realness of the impedance matrix (when velocity excitation is considered). Being $\mathbf{Z}(\omega)=\mathbf{Z}(\omega)^{\mathrm{T}} \in \mathbb{C}^{n \times n}$ the impedance matrix of a generic reciprocal system, with $\mathrm{T}$ denoting the matrix transpose, positive realness implies that $\operatorname{Re}\{\mathbf{Z}(\omega)\} \geq 0 \forall \omega \in \mathbb{R}$. In other words, the real part is positive semidefinite. A real, symmetric matrix $\mathbf{A} \in \mathbb{R}^{n \times n}$ is said to be positive semidefinite if

$$
\mathbf{x}^{\mathrm{T}} \mathbf{A} \mathbf{x} \geq 0
$$

for all non-zero $\mathbf{x} \in \mathbb{R}^{n}$ 35]. To see the correlation between system passivity and positive realness, a multipleinput multiple-output (MIMO) subsystem as the ones presented in Section 2 can be taken as example. At the interface, the time-averaged power transfer by the harmonic forces of complex amplitude $\mathbf{f} \in \mathbb{C}^{n \times 1}$ acting through the velocities of complex amplitude $\mathbf{v} \in \mathbb{C}^{n \times 1}$ is given by:

$$
W(\omega)=\frac{1}{T_{0}} \int_{0}^{T_{0}} \overline{\mathbf{v}}(t) \overline{\mathbf{f}}(t) \mathrm{d} t=\frac{1}{2} \operatorname{Re}\left\{\mathbf{v}^{\mathrm{H}} \mathbf{f}\right\}=\frac{1}{2} \operatorname{Re}\left\{\mathbf{v}^{\mathrm{H}} \mathbf{Z} \mathbf{v}\right\},
$$

where the superscript $\mathrm{H}$ denotes the Hermitian, i.e. the complex conjugate transpose of the vector, $\overline{\mathbf{f}}(t)$ and $\overline{\mathbf{v}}(t)$ the instantaneous forces and velocities while $T_{0}$ is the period of the harmonic vibration. By considering $\mathbf{a}=\operatorname{Re}\{\mathbf{v}\}$ and $\mathbf{b}=\operatorname{Im}\{\mathbf{v}\}$, Eq. (9) can be rewritten as

$$
W=\frac{1}{2} \operatorname{Re}\left\{\{\mathbf{a}-\mathrm{i} \mathbf{b}\}^{\mathrm{T}} \mathbf{Z}\{\mathbf{a}+\mathrm{i} \mathbf{b}\}\right\}=\frac{1}{2}\left(\mathbf{a}^{\mathrm{T}} \operatorname{Re}\{\mathbf{Z}\} \mathbf{a}+\mathbf{b}^{\mathrm{T}} \operatorname{Re}\{\mathbf{Z}\} \mathbf{b}\right) .
$$

The passivity condition $W \geq 0$ is fulfilled when both addends on the right-side of Eq. (10) are non-negative for all possible $\mathbf{a}$ and $\mathbf{b}$. Considering that these two vectors can be arbitrarily chosen and that $\mathbf{Z}$ is symmetric, the passivity condition is met when $\operatorname{Re}\{\mathbf{Z}\}$ is a positive semidefinite matrix. This result implies, inter alia, that the phase angles of the input impedances, i.e. the diagonal elements of the impedance matrix $\mathbf{Z}$, are bound to the interval $\left[-90^{\circ}, 90^{\circ}\right]$.

Several authors over the years have addressed the problem of characterising a physically consistent subsystem through an inverse approach. In the aforementioned study of Carne and Dohrmann [7], a so called conditioning filter is developed to get rid of the non-passive behaviour in an FRF matrix. Considering the mathematical property that a symmetric matrix is positive semidefinite if and only if all its eigenvalues are non-negative, the conditioning filter modifies a synthesised FRF matrix by neglecting the negative eigenvalues. These, however, in case of a highly inconsistent FRF matrix might be large in magnitude and hence carry important information. Their exclusion may consequently lead to substantial changes in the synthesised FRF matrix. Another approach to the problem has been proposed by Sjövall and Abrahamsson [26, 36]. Setting out from input-output data in the frequency domain, a model for both the known subsystem and the assembled system is evaluated in the discrete-time state-space form. In this step, attention is paid to the physical consistency of the evaluated models. Physical properties such as reciprocity and passivity are enforced by performing an unconstrained optimization problem. However, a proportional damping has to be assumed. Once the two known systems are identified, the inverse problem is executed in order to identify the operational interface forces and then characterise the unknown subsystem. In the work presented here a different approach is followed, without the need to transform the input data from the frequency to the state-space domain. In order to enforce reciprocity and passivity conditions, a constrained optimisation scheme has been applied. With this new procedure, no restrictions have to be imposed on the type of system's damping.

\section{Model estimation}

The proposed process to estimate a physically consistent model by means of an inverse approach can be viewed as a three-step procedure.

Firstly, Eq. (4) is solved in a least-squares sense imposing constraints of symmetry and positive real part of the diagonal. This results in a reciprocal dynamic model, which might however not be passive for some frequencies. Subsequently, a conditioning filter modifies the obtained solution in order to guarantee that 
the passivity condition is observed in the full frequency range considered. Finally, a second optimization problem ensures that the modified solution represents the best fit to the input data.

As already mentioned in Section 3, the inverse problem stated in Eq. (4) is usually overdetermined for an improved quality of the results. Hence, it is an unconstrained least-squares problem with a simple analytical solution. If no constraints are imposed, however, a highly inconsistent equivalent impedance matrix is obtained. This is due to the fact that, as already mentioned in Section 3, the behaviour of the subsystem obtained from a continuous interface is condensed into a discrete number of points. In addition, the indirectly evaluation of the interface forces (Eq. (7)) can partially contribute to this inconsistency since the mobility matrix $\mathbf{Y}_{1}$ has no dynamic influence of the continuous interface. The application of the conditioning filter mentioned in Section 4 as a post-processing step would cause substantial changes to the evaluated equivalent impedance matrix, which would not fit any more the original input velocity and force data. Thus, a physically consistent behaviour must be enforced onto the impedance matrix not by post-processing the results but by accurately posing specific constraints to the least-square problem. A constrained least-squares problem must be created, which has no longer a simple analytical solution.

\subsection{The constrained least-squares problem}

A generic constrained least-squares problem can be written in the standard form as:

$$
\begin{aligned}
\operatorname{minimize} & f(\mathbf{x})=\|\mathbf{A} \mathbf{x}-\mathbf{b}\|^{2}=\mathbf{x}^{\mathrm{T}} \mathbf{A}^{\mathrm{T}} \mathbf{A} \mathbf{x}-2 \mathbf{b}^{\mathrm{T}} \mathbf{A} \mathbf{x}+\mathbf{b}^{\mathrm{T}} \mathbf{b} \\
\text { subject to } & c_{k}(\mathbf{x})=0, \quad k=1, \ldots, p \\
& g_{k}(\mathbf{x}) \leq 0, \quad k=1, \ldots, q
\end{aligned}
$$

Here the vector $\mathbf{x} \in \mathbb{R}^{n \times 1}$ is the optimization variable, $\mathbf{A} \in \mathbb{R}^{n \times m}$ is the coefficient matrix and $\mathbf{b} \in \mathbb{R}^{m \times 1}$ the known vector. The function $f: \mathbb{R}^{n} \rightarrow \mathbb{R}$ is the objective function to be minimised and the functions $c_{k}: \mathbb{R}^{n} \rightarrow \mathbb{R}$ together with $g_{k}: \mathbb{R}^{n} \rightarrow \mathbb{R}$ are the equality and inequality constraint functions, respectively. The solution of the problem (11), also known as optimal, is the vector $\mathbf{x}^{*}$ that has the smallest objective value among all vectors that satisfy all the constraints:

$$
\mathbf{x}^{*}=\inf \left\{f(\mathbf{x}) \mid c_{k}(\mathbf{x})=0, k=1, \ldots, p, g_{k}(\mathbf{x}) \leq 0, k=1, \ldots, q\right\} .
$$

The aim is to find the optimal value $\mathbf{x}^{*}$ for the problem $4 \mathbf{Z}_{2}^{e q} \mathbf{V}=\mathbf{F}$, which needs to be transformed in the standard form $\mathbf{A x}=\mathbf{b}$. By keeping in mind that $\mathbf{Z}_{2}^{e q}$ is a symmetric matrix, Eq. (4) is firstly rewritten as:

$$
\mathbf{V}^{\mathrm{T}} \mathbf{Z}_{2}^{e q}=\mathbf{F}^{\mathrm{T}}
$$

and then rearranged as:

$$
\left[\begin{array}{lll}
\ddots & & \\
& {\left[\mathbf{V}^{\mathrm{T}}\right]_{m \times n}} & \\
0 & & \ddots
\end{array}\right]_{m \cdot n \times n \cdot n}\left[\begin{array}{c}
Z_{11} \\
Z_{12} \\
\vdots \\
Z_{1 n} \\
Z_{21} \\
Z_{22} \\
\vdots \\
Z_{n n}
\end{array}\right]_{n \cdot n \times 1}=\left[\begin{array}{c}
F_{11} \\
F_{12} \\
\vdots \\
F_{1 m} \\
F_{21} \\
F_{22} \\
\vdots \\
F_{n m}
\end{array}\right]_{m \cdot n \times 1} \Rightarrow \mathbf{A x}=\mathbf{b}
$$

The coefficient matrix $\mathbf{A} \in \mathbb{C}^{m \cdot n \times n \cdot n}$ contains $n$ times the velocity matrix $\mathbf{V}^{\mathrm{T}}$ in a block diagonal form, with the off-diagonal blocks being zero matrices. The unknown vector $\mathbf{x} \in \mathbb{C}^{n \cdot n \times 1}$ and the known vector $\mathbf{b} \in \mathbb{C}^{m \cdot n \times 1}$ are obtained by stacking the columns of the equivalent impedance matrix and the force matrix transpose on top of each other: $\mathbf{x}=\operatorname{vec}\left(\mathbf{Z}_{2}^{e q}\right), \mathbf{b}=\operatorname{vec}\left(\mathbf{F}^{\mathrm{T}}\right)$. The transformation to the standard form allows to solve the problem in one step, without performing a column by column calculation. Global equality 
and inequality constraints can then be defined and well established optimization algorithms available in the literature can be used.

At this point, proper constraints have to be defined so that the evaluated subsystem model exhibits the correct physical properties. While the property of reciprocity can be easily enforced by imposing symmetry with respect to the main diagonal, the one of passivity needs more attention. As already stated in Section 4. the real part of the equivalent impedance matrix $\operatorname{Re}\left\{\mathbf{Z}_{2}^{e q}\right\}$ must be a positive semidefinite matrix. Hence, all its eigenvalues must be non-negative. This condition cannot be enforced without the use of non-linear inequality constraints, which makes the optimization problem difficult to solve. Instead, linear inequality constraints that enforce the diagonal elements of $\operatorname{Re}\left\{\mathbf{Z}_{2}^{e q}\right\}$ to be non-negative are applied in a first step. For this purpose, a quadratic program, i.e. an optimization problem having a quadratic objective function and affine constraints functions, is used. While non-linear optimisation always involves some kind of compromise, such as very long computation times or even the risk of not finding a solution at all, a quadratic program can be solved very reliably and efficiently, using interior-point methods or other special methods for convex optimization [37.

In order to insert inequality constraints only on the real part of the solution, the problem is rewritten by separating the real and imaginary data:

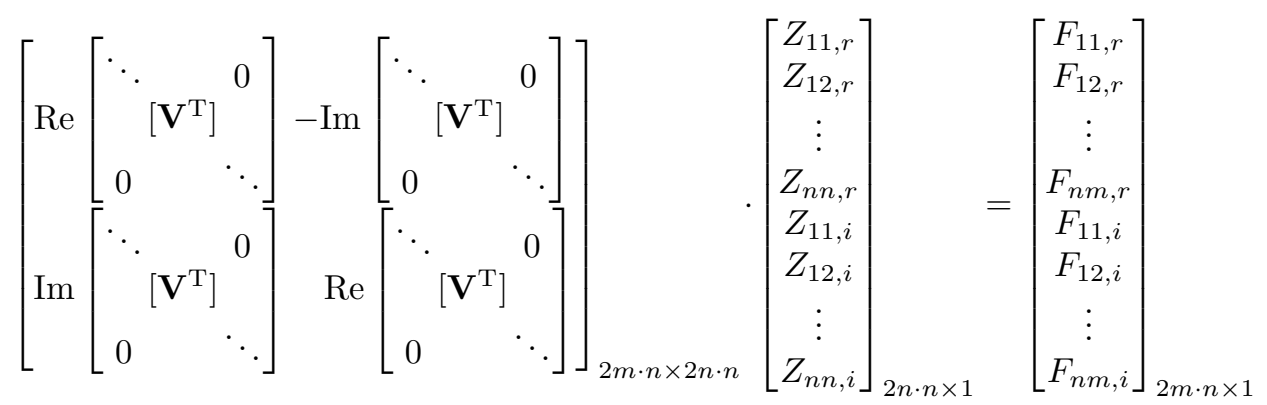

where the subscripts $r$ and $i$ denote the real and imaginary part of the complex quantities. Eq. (15) is a real least-squares problem with $\mathbf{A} \in \mathbb{R}^{2 m \cdot n \times 2 n \cdot n}, \mathbf{x} \in \mathbb{R}^{2 n \cdot n \times 1}$ and $\mathbf{b} \in \mathbb{R}^{2 m \cdot n \times 1}$.

Constraints can now be defined, and the constrained least-squares problem (11) becomes:

$$
\begin{aligned}
\operatorname{minimize} & f(\mathbf{x})=\|\mathbf{A} \mathbf{x}-\mathbf{b}\|^{2}=\mathbf{x}^{\mathrm{T}} \mathbf{A}^{\mathrm{T}} \mathbf{A} \mathbf{x}-2 \mathbf{b}^{\mathrm{T}} \mathbf{A} \mathbf{x}+\mathbf{b}^{\mathrm{T}} \mathbf{b} \\
\text { subject to } & \mathbf{C} \mathbf{x}=0 \\
& \mathbf{G} \mathbf{x} \leq 0
\end{aligned}
$$

$\mathbf{C} \in \mathbb{R}^{2 p \times 2 n \cdot n}$ and $\mathbf{G} \in \mathbb{R}^{n \times 2 n \cdot n}$ are signed Boolean matrices properly built to enforce the aforementioned conditions:

$$
\begin{aligned}
Z_{i j, \beta} & =Z_{j i, \beta} \\
-Z_{i i, r} & \leq 0,
\end{aligned}
$$

with $i, j \in\{1, \ldots, n\}$ and $\beta \in\{r, i\}$. In total, $p$ equality constraints and $n$ inequality constraints are defined, with $p=n(n-1)$. Problem $(16)$ is solved in the Python-embedded modeling language for convex optimization problems CVXPY 38. The open source, interior-point solver ECOS (embedded conic solver) [39] is adopted. Interior-point methods tackle an inequality constrained problem by solving a sequence of unconstrained, or equality constrained, problems. The optimal solution is iteratively approached from the interior of the feasible set, without tending to favour solutions that are at the border of the feasible region. Details regarding the interior-point method are given by Boyd and Vandenberghe [37.

\subsection{Conditioning filter}

The optimization problem 16 gives as result $\mathbf{Z}_{r}$ and $\mathbf{Z}_{i}$, i.e. real and imaginary part of the impedance matrix $Z$

$$
\mathbf{Z}=\mathbf{Z}_{r}+\mathrm{i} \mathbf{Z}_{i}
$$


$\mathbf{Z}$ represents the best fit of the coupled velocity $\mathbf{V}$ and force $\mathbf{F}$ data given as input under the constraints of symmetry and real semi-positiveness of the diagonal entries. These are however necessary but not sufficient conditions for a matrix to have a positive semi-definite real part. An impedance matrix which does not completely fulfil the passivity requirement might be obtained. Hence, the implementation of a filtering technique as the one mentioned in Section 4 will remove non-physical behaviours and ensures that the passivity condition is met. The filter acts on the real part of the impedance matrix $\mathbf{Z}_{r}$, which can be rewritten using an eigenvalue decomposition as:

$$
\mathbf{Z}_{r}=\phi \lambda \phi^{\mathrm{T}}
$$

The columns of $\phi \in \mathbb{R}^{n \times n}$ are the eigenvectors of $\mathbf{Z}_{r}$ while the diagonal matrix $\boldsymbol{\lambda} \in \mathbb{R}^{n \times n}$ contains the corresponding eigenvalues. In order for $\mathbf{Z}_{r}$ to be a positive semi-definite matrix, none of the eigenvalues shall be negative. Hence, a new diagonal matrix $\tilde{\lambda}$ is built by setting all the negative eigenvalues equal to zero:

$$
\tilde{\lambda}_{i i}=\left\{\begin{array}{ll}
\lambda_{i i}, & \text { if } \lambda_{i i} \geq 0 \\
0, & \text { if } \lambda_{i i}<0
\end{array}, \quad \text { with } i \in\{1, \ldots, n\} .\right.
$$

The filtered impedance matrix $\tilde{\mathbf{Z}}_{r}$ is then given by:

$$
\tilde{\mathbf{Z}}_{r}=\phi \tilde{\lambda} \phi^{\mathrm{T}} .
$$

All the eigenvalues of $\tilde{\mathbf{Z}}_{r}$ are greater or equal to zero and, hence, $\tilde{\mathbf{Z}}_{r}$ is a positive semi-definite matrix. The passivity condition for the equivalent impedance matrix is therefore satisfied.

\subsection{Second optimization step}

The drawbacks introduced by the filtering procedure are, however, twofold. Not only the information carried by the omitted eigenvalues is lost and hence approximations are introduced but on top of this, being $\mathbf{Z}_{r}$ modified by the filter, is not anymore the least-squares solution for the problem (4) $\mathbf{Z}_{2} \mathbf{V}=\mathbf{F}$. It is however assumed that $\mathbf{Z}_{r}$, after the constrained optimization, is close to be positive semi-definite. If some eigenvalues result to be negative, their amplitude is assumed to be small as compared to the dominant eigenvalues. Thus, it is assumed that they do not carry relevant information. The second issue is solved by performing a second optimization problem. The real part of the impedance matrix $\tilde{\mathbf{Z}}_{r}$ is now fixed and the imaginary part $\mathbf{Z}_{i}$ is again evaluated. By solving this problem, an equivalent impedance matrix which represents the least-squares solution for the problem (4) under the constraint of physical consistency is obtained. In order to re-evaluate $\mathbf{Z}_{i}$ having as input the matrices $\mathbf{V}, \mathbf{F}$ and $\tilde{\mathbf{Z}}_{r}$, the matrix equation 15 is rewritten as:

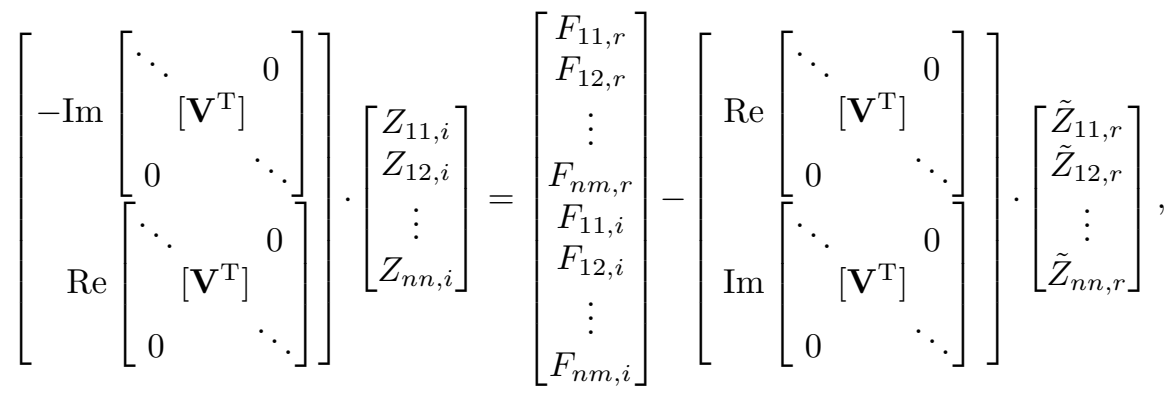

where the real part $\tilde{\mathbf{Z}}_{r}$ together with the corresponding columns of the coefficient matrix $\mathbf{A}$ are moved to the right-hand side. This problem has a number $n$ of unknowns and does not need any inequality constraint. The imaginary part of the impedance matrix is indeed not directly involved in the passivity condition and, therefore, only the property of symmetry must be satisfied. An equally constrained least-squares problem is created:

$$
\begin{aligned}
\text { minimize } & f(\mathbf{x})=\|\mathbf{A} \mathbf{x}-\mathbf{b}\|^{2}=\mathbf{x}^{\mathrm{T}} \mathbf{A}^{\mathrm{T}} \mathbf{A} \mathbf{x}-2 \mathbf{b}^{\mathrm{T}} \mathbf{A} \mathbf{x}+\mathbf{b}^{\mathrm{T}} \mathbf{b} \\
\text { subject to } & \mathbf{C} \mathbf{x}=0
\end{aligned}
$$


where $\mathbf{A} \in \mathbb{R}^{2 m \cdot n \times n \cdot n}, \mathbf{x} \in \mathbb{R}^{n \cdot n \times 1}$ and $\mathbf{b} \in \mathbb{R}^{2 m \cdot n \times 1}$ are the coefficient matrix, the unknown and known vectors of 22 , respectively. $\mathbf{C} \in \mathbb{R}^{p \times n \cdot n}$ is a signed Boolean matrix which enforces the condition:

$$
Z_{i j, i}=Z_{j i, i} .
$$

A direct solution for this problem can be found using a Lagrangian approach. The basic idea is to take the constraints in (23) into account by augmenting the objective function with a weighted sum of the constraint functions. The so-called Lagrangian function $\mathcal{L}: \mathbb{R}^{n} \times \mathbb{R}^{p} \rightarrow \mathbb{R}$ associated with the problem 23] is defined as

$$
\mathcal{L}(\mathbf{x}, \boldsymbol{\lambda})=f(\mathbf{x})+\sum_{k=1}^{p} \lambda_{k} \mathbf{c}_{k} \mathbf{x},
$$

where $\lambda_{k}$ is the Lagrange multiplier associated with the $k$ th equality constraint $\mathbf{c}_{k} \mathbf{x}=0$, being $\mathbf{c}_{k}$ the $k$ th row of the matrix $\mathbf{C}$ in $(23)$. The optimality condition is found by solving the problem

$$
\nabla_{\mathbf{x}, \boldsymbol{\lambda}} \mathcal{L}(\mathbf{x}, \boldsymbol{\lambda})=0
$$

which forms the system equations

$$
\left[\begin{array}{cc}
2 \mathbf{A}^{\mathrm{T}} \mathbf{A} & \mathbf{C}^{\mathrm{T}} \\
\mathbf{C} & 0
\end{array}\right] \cdot\left[\begin{array}{l}
\mathbf{x} \\
\boldsymbol{\lambda}
\end{array}\right]=\left[\begin{array}{c}
2 \mathbf{A}^{\mathrm{T}} \mathbf{b} \\
0
\end{array}\right] .
$$

This square set of $n+p$ linear equations in the variables $\mathbf{x}, \boldsymbol{\lambda}$ is also known as the Karush-Kuhn-Tucker $(K K T)$ conditions. Given the nature of both objective function and constraints, the problem (27) always admits a solution $\left(\mathbf{x}^{*}, \lambda^{*}\right)$, which represents a saddle point of the Lagrangian $\mathcal{L}$ [37. This solution is the imaginary part of the impedance matrix $\mathbf{Z}_{i}$, which combined with the real part $\tilde{\mathbf{Z}}_{r}$ leads to an impedance matrix that represents the best fit of the input velocity and force data under the constraints of passivity and reciprocity.

\subsection{Method overview}

A schematic overview of the proposed approach is given in Figure 2. By solving the least-squares problem (4) for the frequency line $\hat{\omega}$, real and imaginary parts of $\mathbf{Z}_{2}^{e q}$ are evaluated under the constraints of symmetry and real semi-positiveness of the diagonal entries. These constraints represent necessary but not sufficient conditions for passivity. A check for semi-positiveness of the real part $\operatorname{Re}\left\{\mathbf{Z}_{2}^{e q}\right\}$ is therefore done by looking at its eigenvalues. If all the eigenvalues result to be non-negative, the solution is accepted. If not, $\operatorname{Re}\left\{\mathbf{Z}_{2}^{e q}\right\}$ is adjusted by filtering out the negative eigenvalues. The optimal solution under the new constraints is re-evaluated with a new least-squares solution, where the filtered $\operatorname{Re}\left\{\mathbf{Z}_{2}^{e q}\right\}$ is given as input together with the force and velocity matrices $\mathbf{F}$ and $\mathbf{V}$. An impedance matrix which expresses the best fit of the coupled system under the constraints of reciprocity and passivity is finally obtained. This process is then repeated for all the frequency lines of interest.

\subsection{Result validation}

As already mentioned in Section 3 , the validation of the obtained equivalent impedance matrix cannot be carried out by comparison with the impedance matrix directly evaluated on the stand-alone component. A direct characterisation, starting from velocity and force data at the coupling points of the stand-alone subsystem, would lead to an impedance matrix which does not include the effect of the continuous interface. An alternative approach to verify the accuracy of the proposed method is to validate by reassembly. The characterised receiver subsystem is coupled with the source subsystem via the superposition principle, i.e. solving the equation system given by the combination of equations (3) and (6):

$$
\left[\begin{array}{cc}
\mathbf{Y}_{1} & \mathbf{I} \\
\mathbf{I} & -\mathbf{Z}_{2}^{e q}
\end{array}\right] \cdot\left[\begin{array}{l}
\mathbf{f}_{c} \\
\mathbf{v}_{c}
\end{array}\right]=\left[\begin{array}{c}
\mathbf{f}_{S} \cdot \mathbf{y}_{S} \\
0
\end{array}\right]
$$




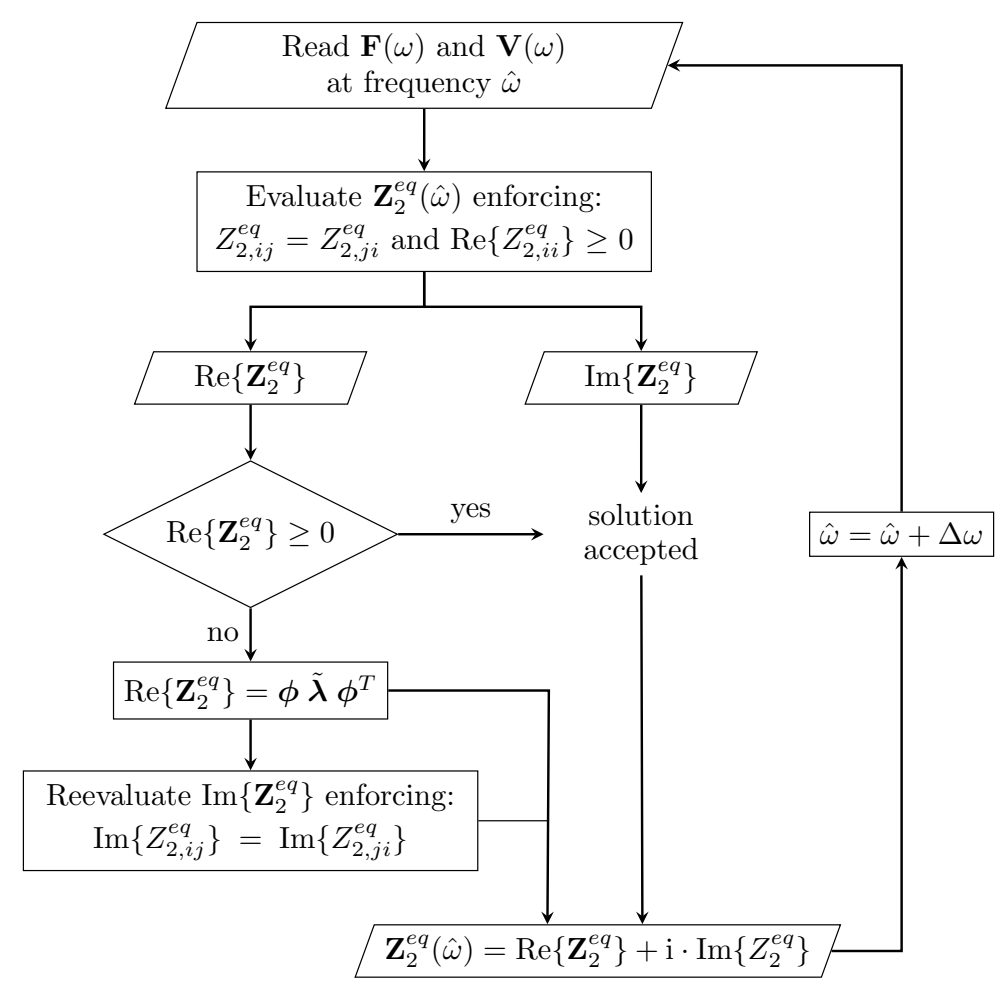

Figure 2: workflow of the proposed approach to inversely evaluate a physically consistent impedance matrix.

where $\mathbf{I}$ is an identity matrix of size $n$ and $\mathbf{y}_{S}$ the source transfer function, i.e. the velocity at the coupling points when a unitary force is applied to the source location. The solution of the set of equations (28) gives as result the forces $\mathbf{f}_{c}$ and velocities $\mathbf{v}_{c}$ at the coupling points due to the interaction of the receiver with the source subsystem, excited by the input force $\mathbf{f}_{S}$. Subsequently, the transfer functions $\mathbf{y}_{R}$ are obtained by successively exciting a single coupling DoF with a unitary force and measuring the velocity response at the receiving position. Their product with the coupled forces $\mathbf{f}_{c}$ gives the sought velocity response $\mathbf{v}_{R}$ :

$$
\mathbf{v}_{R}=\mathbf{y}_{R} \mathbf{f}_{c} .
$$

Finally, $\mathbf{v}_{R}$ is compared with the reference velocity obtained with the fully coupled system, hence having the two subsystems continuously connected at their common interface.

\section{Example}

In this section, the proposed method is applied to a beam-plate FE system. NX Nastran [40] has been used as FE solver while all the calculations have been done in a Phyton 41] environment.

\subsection{System description}

Figure 3 shows a system made of two horizontal plates connected along a common edge via an hollow beam. Both plates and beam are free to move and have the same characteristics, given in Table 1 . The plates lie in the $z=0$ plane and are, together with the beam, discretised with an FE mesh fine enough to meet the "6 elements per bending wavelength" criterion in the considered frequency range. One layer of quadrilateral isotropic shell elements CQUAD4 is chosen. The first plate together with the beam constitutes the source subsystem, which as shown in Figure 3 is excited by an out-of-plane force at point $S$. The second plate represents the receiver subsystem, which is passive. Two receiving points $R_{1}$ and $R_{2}$, respectively on 


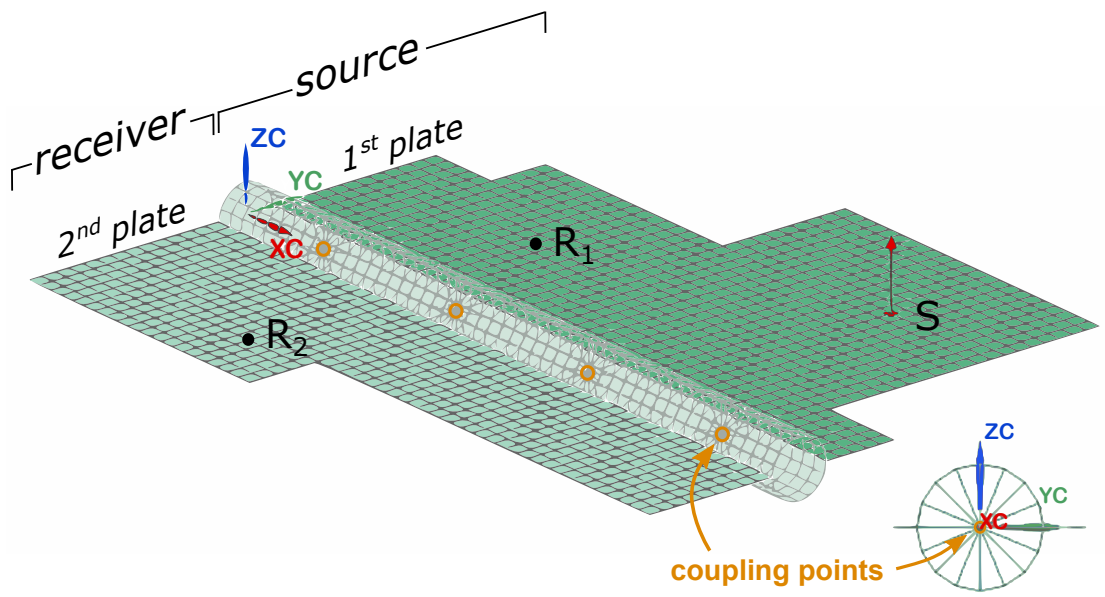

Figure 3: Beam-plate system.

Table 1: System's material and geometric characteristics.

\begin{tabular}{lccc}
\hline \hline Parameter & Notation & Value & Unit \\
\hline & & & \\
Young's modulus & $\mathrm{E}$ & 70 & $\mathrm{GPa}$ \\
Poisson coefficient & $\nu$ & 0.3 & - \\
Density & $\rho$ & 2700 & $\mathrm{~kg} \mathrm{~m}^{-3}$ \\
Structural damping coefficient & $\eta$ & 0.05 & - \\
Length of the interface & $\mathrm{L}$ & 0.5 & $\mathrm{~m}$ \\
Thickness & $\mathrm{h}$ & 0.004 & $\mathrm{~m}$ \\
\hline
\end{tabular}

the source and receiver subsystem, are considered. The whole beam is considered here as interface. This translates to the fact that the coupling points used to discretise its dynamic behaviour are located on its axis and not on the seam between the 2nd plate and the side of the beam. Consequently, local distortions due to a punctual excitation of the beam's edge during the evaluation of the interface FRFs cannot occur. The coupling points are consequently connected through rigid RBE2 elements to the nodes within the same cross-section, as shown in the beam's profile in the bottom right corner of Figure3. The load applied to evaluate the interface FRFs is then uniformly spread to the whole (rigid) cross-section of the beam. 12 coupling DoFs are considered in total, i.e. the out-of-plane translational DoF and the in-plane rotational DoFs (bending rotational DoFs around the axis in the plane of the plate) for each coupling point. It is worth mentioning that, since the FE model contains 51 nodes at the interface, by considering the point discretisation the interface problem size is reduced by a factor of about 13 .

\subsection{Results}

To apply the inverse approach presented in this paper, the matrices of coupled velocities $\mathbf{V}$ and forces $\mathbf{F}$ should be created. The source subsystem is then coupled with the receiver and excited with a single out of plane force in 24 arbitrarily chosen positions. Their random position together with the asymmetric shape of the system plates are such to create a linearly independent set of load cases, so that system (16) will be overdetermined by a factor of 2 . For each load case, coupled velocities $\mathbf{v}_{c}$ are calculated and stored as columns in the matrix $\mathbf{V}$. Attention is paid that none of the load cases is located in the source position $S$ or in its neighbourhood, such that the excitation used to test the model does not coincide with one of the load cases used to build the model itself. The force matrix $\mathbf{F}$ is indirectly evaluated as described in Section 3.2 
Mobility matrix $\mathbf{Y}_{1}$ and free velocities $\mathbf{v}_{\text {free }}$ (see Eq. (7) ) are obtained through direct calculation on the disconnected source subsystem. For each load case, free velocities $\mathbf{v}_{\text {free }}$ are calculated and stored as column in the matrix $\mathbf{V}_{\text {free }}$. The matrix of coupled forces $\mathbf{F}$ can be now evaluated according to equation (7).

Once the force and velocity matrices are obtained, the receiver impedance matrix $\mathbf{Z}_{2}^{e q}$ is inversely evaluated by following the process described in Figure 2. Firstly, the impedance matrix is evaluated by solving the constrained least-squares problem (16). The imposed constraints are crucial in order to enforce a physical behaviour. Figure 4 presents some impedance matrix elements obtained with the straightforward leastsquares approach, that is, without imposing constraints. The matrix turns out to be non-symmetric and negative real parts occur in the diagonal entries. It means that the receiver subsystem is not reciprocal and
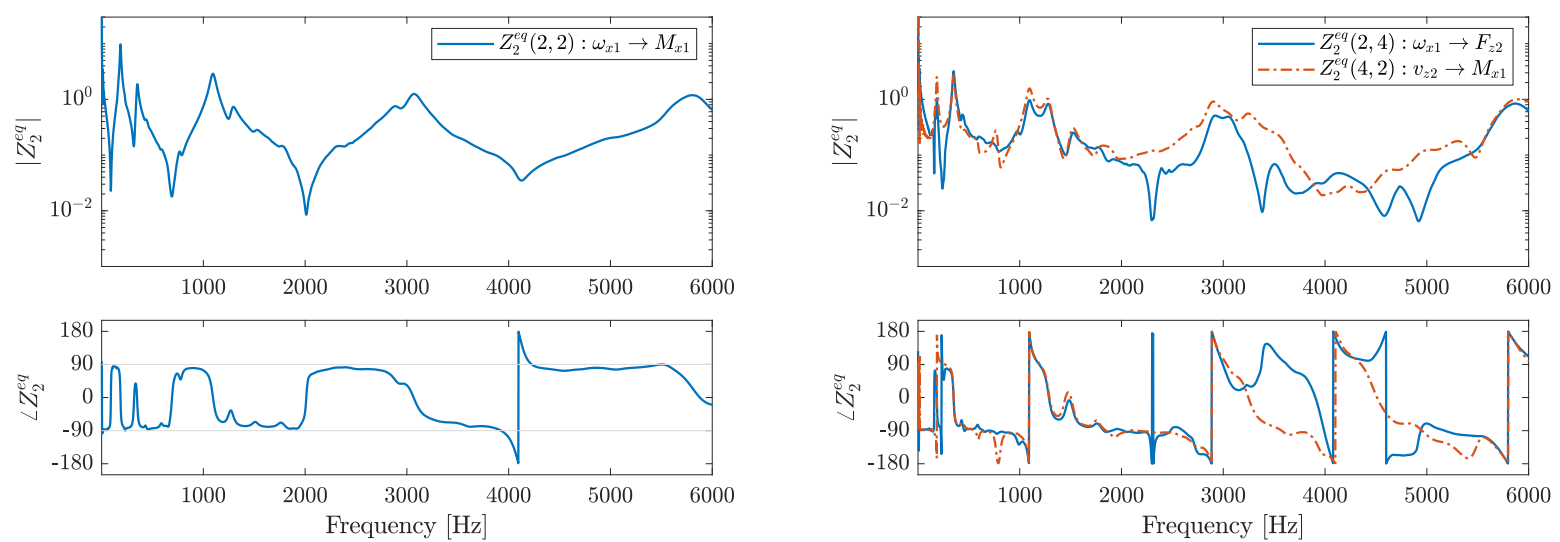

(a) Input impedance $\mathbf{Z}_{2}^{e q}(2,2)$

(b) Cross transfer impedances $\mathbf{Z}_{2}^{e q}(2,4)$ and $\mathbf{Z}_{2}^{e q}(4,2)$

Figure 4: Input impedance for the 2 nd DoF, i.e. the x-rotation of coupling point 14 and cross transfer impedances relating the 2 nd and 4 th DoFs, i.e. the x-rotation of coupling point 1 and z-displacement of coupling point 2 4b, when no constraints are imposed to the least-squares problem.

passive as it should be. On the contrary, the same elements of $\mathbf{Z}_{2}^{e q}$ evaluated with the constraints stated in (17) are presented in Figure 5 and show physical consistency. The two types of constraints imposed,
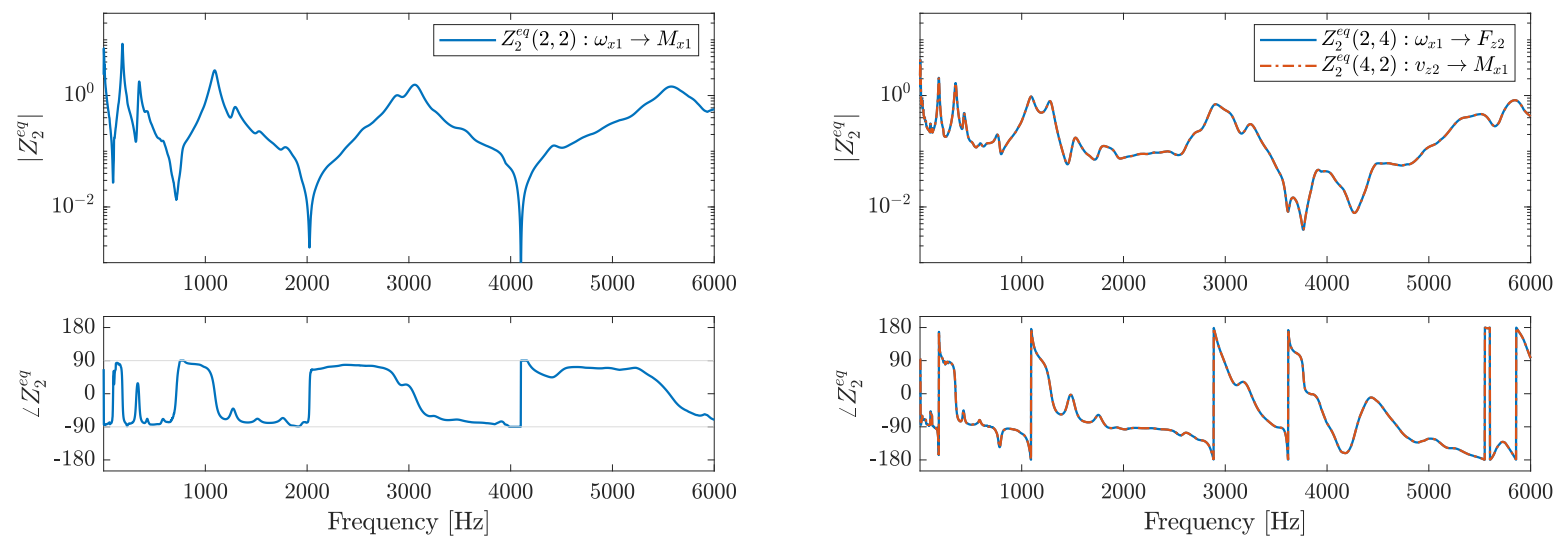

(a) Input impedance $\mathbf{Z}_{2}^{e q}(2,2)$

(b) Cross transfer impedances $\mathbf{Z}_{2}^{e q}(2,4)$ and $\mathbf{Z}_{2}^{e q}(4,2)$

Figure 5: Input impedance for the 2nd DoF $5 \mathrm{a}$ and cross transfer impedances relating the 2nd and 4th DoFs $5 \mathrm{~b}$ when appropriate constraints are imposed to the least-squares problem. 
however, are not sufficient to ensure positive semi-definiteness to the real part of the impedance matrix $\operatorname{Re}\left\{\mathbf{Z}_{2}^{e q}\right\}$. Figure 6 plots the 12 eigenvalues of $\operatorname{Re}\left\{\mathbf{Z}_{2}^{e q}\right\}$ obtained with the constrained least-squares problem. It can be observed how some eigenvalues become negative for specific frequency ranges even though

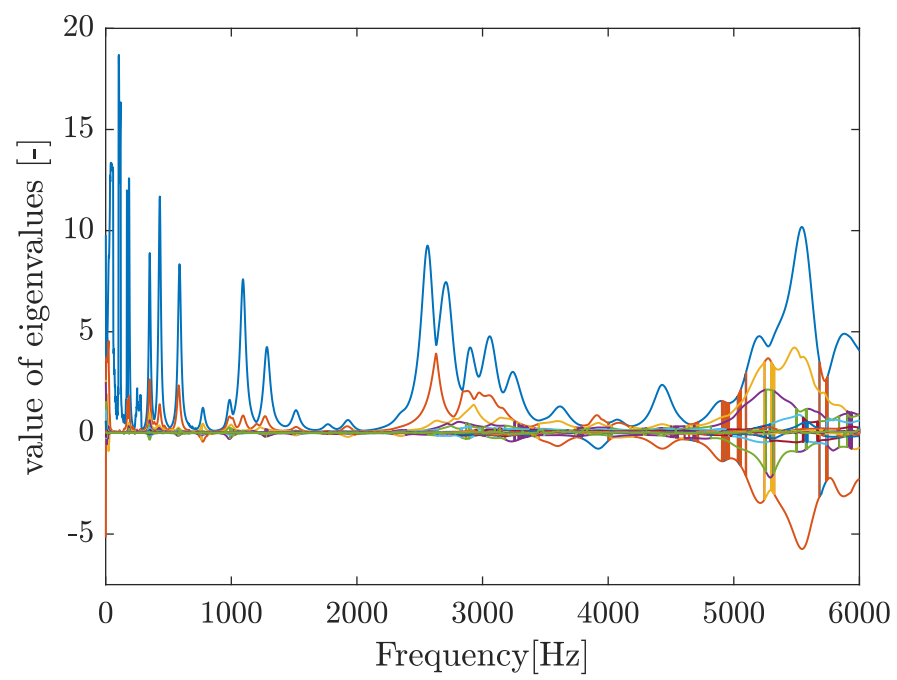

Figure 6: Eigenvalues of the real part of the evaluated impedance matrix $\operatorname{Re}\left\{\mathbf{Z}_{2}^{e q}\right\}$ versus frequency. A different colour is assigned to each of the 12 eigenvalues. (For interpretation of the references to colour in this figure, the reader is referred to the web version of this article.)

the impedance matrix is symmetric with the real part of the diagonal elements non-negative. This proves that the imposed constraints are necessary, though not sufficient, to enforce positive semi-definiteness to $\operatorname{Re}\left\{\mathbf{Z}_{2}^{e q}\right\}$. However, they lead to a matrix which is close to be positive semi-definite until $f_{\text {lim }} \simeq 4500 \mathrm{~Hz}$. Indeed, as it can be noticed in Figure 6, up to this frequency the negative eigenvalues are small compared to the largest at any particular frequency, meaning that they carry less relevant information with respect to the latter. By neglecting them with the conditioning filter described in Section 5.2, the elements of the impedance matrix do not change substantially. Above $f_{l i m}$, the presence of dominant negative eigenvalues shows the difficulties of the optimisation problem in finding a solution which fits the input data while complying with the requirements of physical consistency. After the application of the conditioning filter, the new impedance matrix does not represent the least-squares solution for the optimization problem (16) any more. To ensure that at the end of the process the best solution which fulfils the physical requirements is taken, the second optimization step described in Section 5.3 is carried out. Figure 7 shows how up to $f_{\text {lim }}$ the impedance matrix is slightly changing after the implementation of the conditioning filter together with the second optimization step. Above this frequency, substantial changes are detected. The negative eigenvalues which have been neglected are, indeed, comparable in magnitude with the positive ones which have been retained.

The physically consistent equivalent impedance matrix is finally used in the coupling procedure described in Section 5.5. Firstly, the coupling forces $\mathbf{f}_{c}$ are evaluated by applying the superposition principle. The coupling forces, together with the receiver transfer functions, allow the evaluation of the out-of-plane velocity at a generic point on the first and second plate. These are compared with a reference solution, obtained by considering the fully coupled system. As shown in Figure 8, the proposed approach to indirectly evaluate a physically consistent impedance matrix leads to a good reconstruction of the dynamic behaviour of the coupled system. The results fit well approximatively up to $f_{\text {lim }}$ when the receiving location is on the receiver subsystem (Figure 8b). Above this frequency, the chosen number of coupling points is not sufficient to properly describe the dynamic phenomena occurring at the interface. In other words, the number of discretised forces and velocities are not enough to build a physically consistent equivalent impedance matrix that correctly approximates the dynamics of the receiver subsystem when coupled via a continuous interface. 

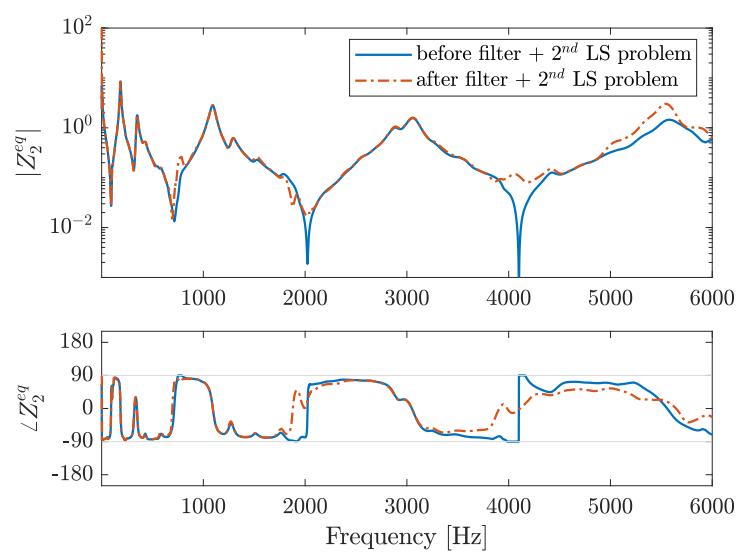

(a) Input impedance $Z_{2}^{e q}(2,2)$
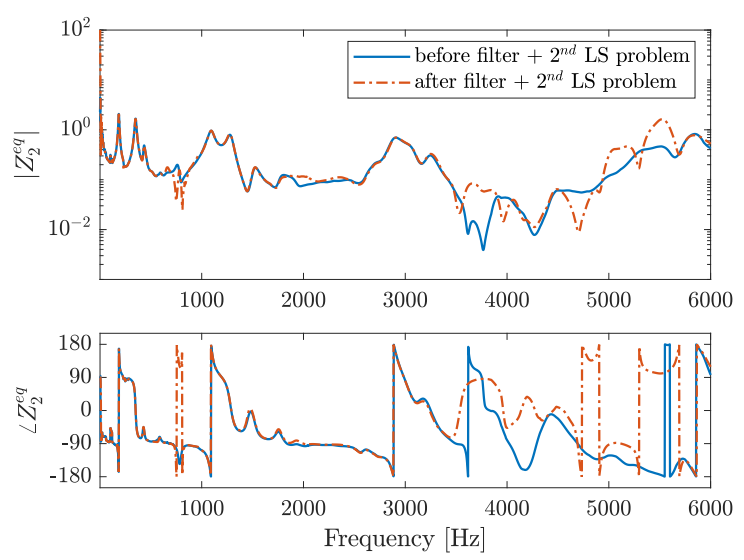

(b) Cross transfer impedances $Z_{2}^{e q}(2,4)$ and $Z_{2}^{e q}(4,2)$

Figure 7: Input impedance for the 2nd DoF $7 \mathrm{a}$ and cross transfer impedances relating the 2nd and 4th DoFs $7 \mathrm{~b}$. The application of the conditioning filter together with the 2nd optimisation step enforce the passivity property without bringing consistent changes to the impedance matrix.

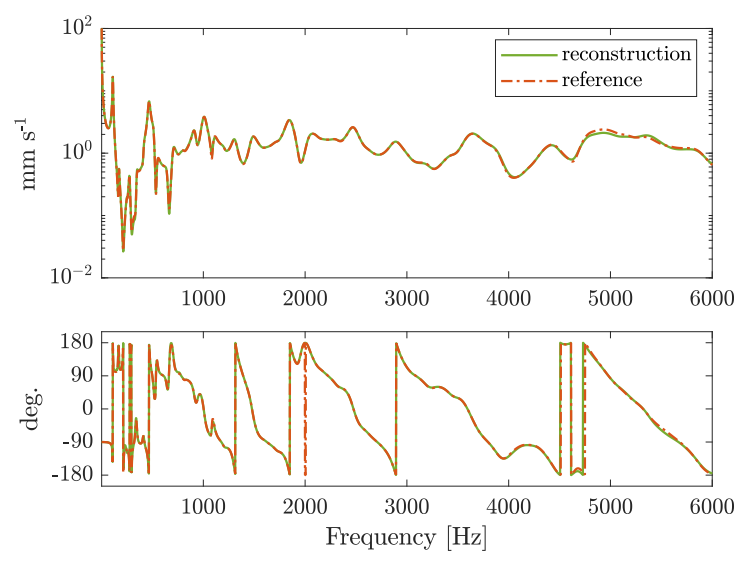

(a) out-of-plane velocity at $R_{1}$
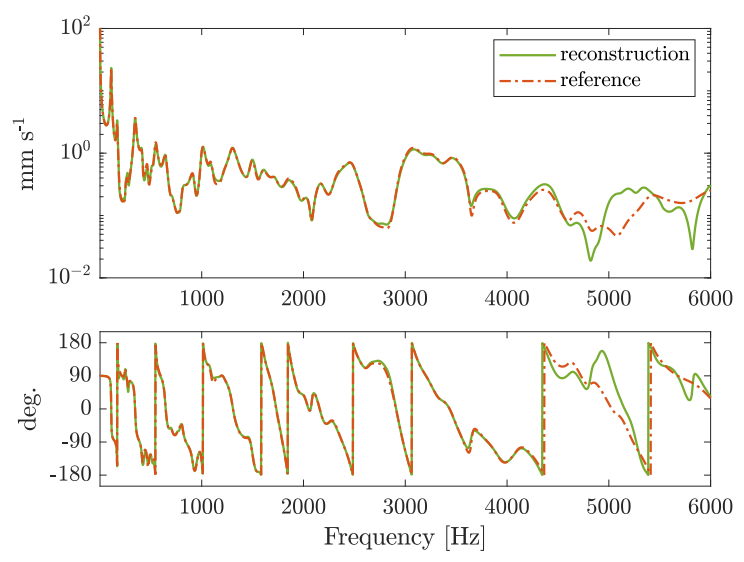

(b) out-of-plane velocity at $R_{2}$

Figure 8: Out-of-plane velocity of a point located on the source $8 \mathrm{a}$ and receiver $8 \mathrm{~b}$ subsystem. The reconstruction evaluated with the substructuring approach is compared with the reference, evaluated using the fully coupled system.

Less pronounced differences are noticeable above $f_{\text {lim }}$ for the case when the receiving location is on the source subsystem (Figure 8a), probably due to the little coupling influence that the receiver has on the source in that frequency range.

\subsection{Modified system}

In the previous section, the accuracy of the proposed methodology has been tested by analysing the dynamic behaviour of the coupled system. The same coupled system has however been used for the evaluation of the equivalent impedance matrix. In order to strengthen the validation of the proposed methodology, the inversely characterised receiver subsystem is attached to a new source (Figure 9), which does not represent any more a "friendly" subsystem. The new system is excited by an out-of-plane force at point $S$. Two receiving points $R_{1}$ and $R_{2}$, respectively on the source and receiver subsystem, are again considered. The coupling procedure described in Section 5.5 is repeated and the out-of-plane velocity at the receiving location 


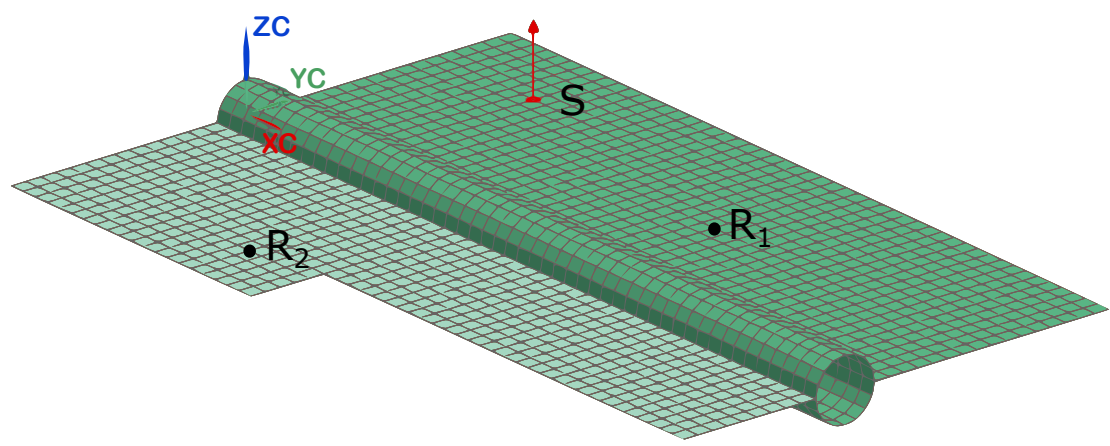

Figure 9: Beam-plate system modified with a new different source substructure.

evaluated. The results presented in Figure 10 show a good correlation between reference and substructuring reconstruction approximatively up to the same $f_{\text {lim }}$ above which the number of negative eigenvalues explodes. Since the interface between the plates consists of a relatively stiff beam, the governing wavelength can be approximated by that of the stand-alone beam which turns out to be about $300 \mathrm{~mm}$ at $4500 \mathrm{~Hz}$. This approximately corresponds with 2 times the spacing of the coupling points $(125 \mathrm{~mm})$, suggesting that the Nyquist-Shannon sampling criterion can also be used for the discretisation of the interface and providing a physical interpretation to $f_{\text {lim }}$. Comparable results have been shown by Meggitt and Moorhouse [31] and Meggit et al. [42, where the concept of interface completeness, i.e. the quality of the interface description whilst considering a reduced set of interface DoFs, is assessed on a rectangular plate separated in two sides by a line.

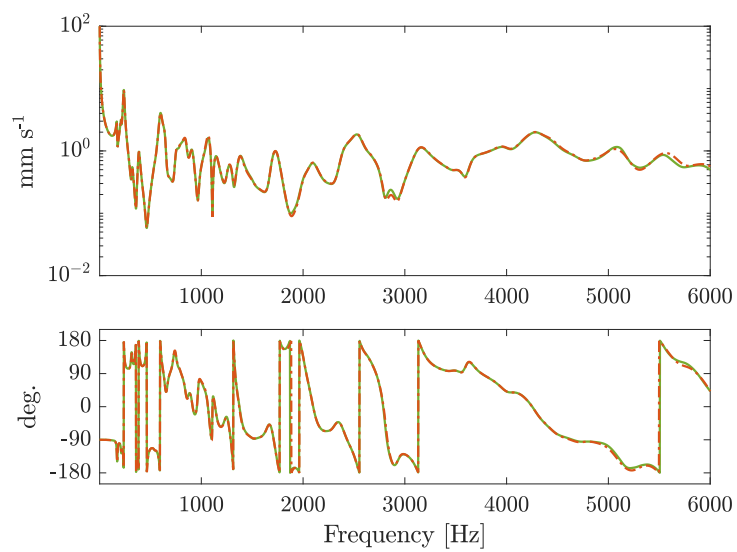

(a) out-of-plane velocity at $R_{1}$
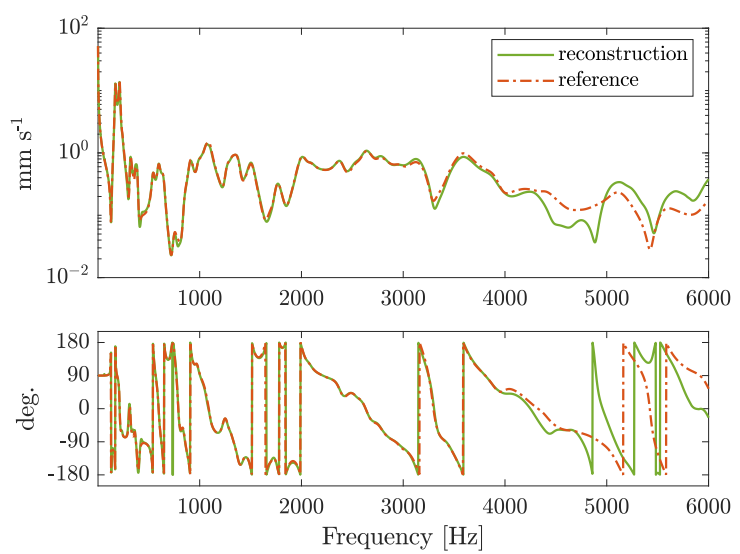

(b) out-of-plane velocity at $R_{2}$

Figure 10: Out-of-plane velocity of a point located on the source $10 \mathrm{a}$ and receiver $10 \mathrm{~b}$ subsystem. The reconstruction evaluated with the substructuring approach is compared with the reference, evaluated using the fully coupled system.

\subsection{System with noisy data}

In order to test its robustness and reliability, the presented method is validated using noisy data. The objective is to investigate how the three-step approach to enforce physical consistency of inversely characterised subsystems is behaving when the lack of reciprocity and passivity is not only caused by methodological and modelling errors. Artificial "measurement noise" is therefore added to the system. The elements of the noisy 
velocity matrix $\overline{\mathbf{V}}$ (and analogously of the force matrix $\overline{\mathbf{F}}$ ) at the discrete frequencies $\omega_{k}$ are constructed as:

$$
\overline{\mathbf{V}}_{i j}\left(\omega_{k}\right)=\mathbf{V}_{i j}\left(\omega_{k}\right)+\alpha_{i j k}+\mathrm{i} \beta_{i j k}
$$

where $\alpha_{i j k}$ and $\beta_{i j k}$ are Gaussian distributed independent stochastic variables with zero mean and standard deviations of $3 \cdot 10^{-3} \mathrm{~mm} \mathrm{~s}^{-1}$ and $2 \cdot 10^{-3} \mathrm{~N}$, respectively. Two elements of these noisy matrices are shown in Figure 11. Next the three-step approach is repeated using these noisy matrices and a new equivalent
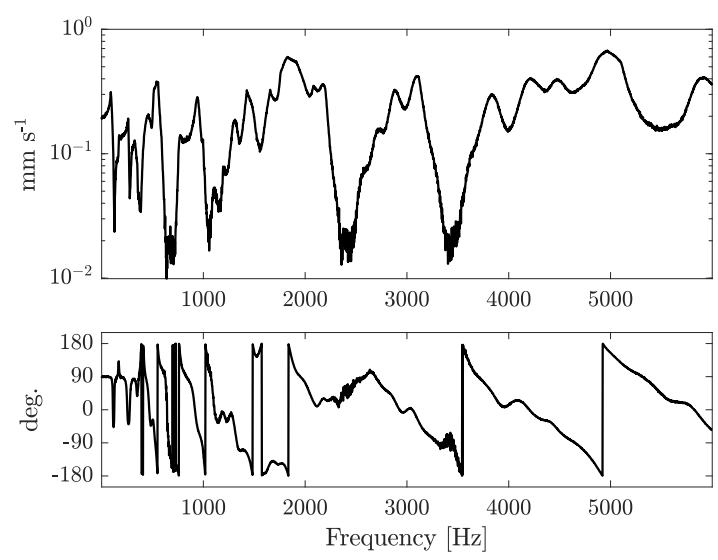

(a) noisy velocity $V(6,14)$
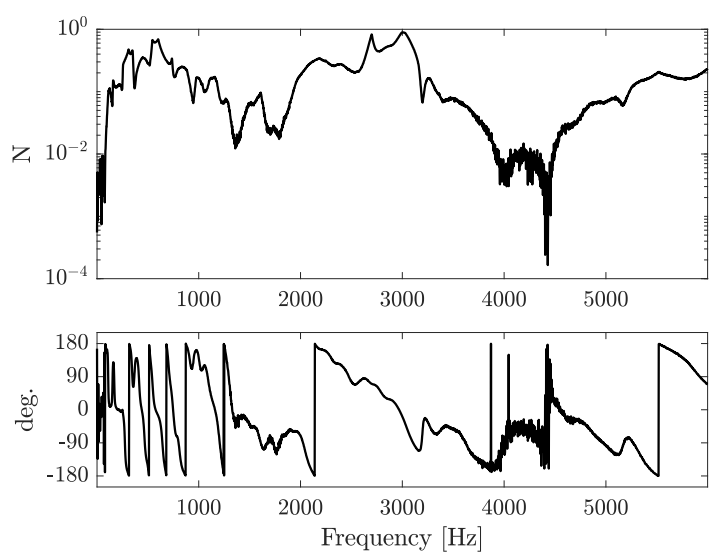

(b) noisy force $F(8,24)$

Figure 11: Elements of the velocity $\sqrt{11 \mathrm{a}}$ and force $11 \mathrm{~b}$ matrix after the introduction of artificial noise.

impedance matrix $Z_{2}^{e q}$ is obtained as output. One of its diagonal elements is displayed in the form of blue dots in Figure 12a showing, as expected, a noisy behaviour. However, the obtained impedance matrix again turns out to be physically consistent. In order to highlight this fact, the corresponding impedance curve resulting from the un-constrained solution of the inverse problem (4) was added to Figure 12a In can be observed that the proposed three-step method indeed removes the unphysical behaviour enforcing phase angles within the interval $[-90,90]$. By comparing Figure 12a with Figure 4a it can further be observed that the unphysicalities below $3000 \mathrm{~Hz}$ were introduced by the noise pollution. This suggests that the non-physical behaviour caused by external phenomena such as noise can be successfully removed using the proposed methodology. Once its physical consistency is assured, it is important to verify up to which extent the noisy impedance matrix varies from the one previously obtained with clean data as input. A simple moving average with 20 samples is then considered for the physical input mobility of Figure 12a, which is then compared in Figure $12 \mathrm{~b}$ with the corresponding physical input mobility evaluated beforehand in absence of noise. Also for the latter a moving average is considered for the sake of comparability. A good agreement is obtained indicating that the 3 -step procedure applied to noisy data did not affect the average behaviour of the impedance curve. The noisy impedance matrix is afterwards used in the coupling scheme of Equation (28), where also the other FRFs were polluted with a Gaussian distribution having zero mean and standard deviations of $3 \cdot 10^{-3} \mathrm{~mm} \mathrm{~s}^{-1} \mathrm{~N}^{-1}$. The out-of-plane velocity at the receiving locations is subsequently evaluated and, as presented in Figure 13, is in accordance with the previous "unnoisy" results. A good reconstruction is obtained approximatively up to the $f_{\text {lim }}$ defined and discussed above. Substantial changes are detected above this frequency, especially for the output velocity at the receiver plate, which results to be more affected by the noisy FRF matrices.

\section{Conclusions}

Inverse methods to retrieve the impedance matrix of a subsystem from the coupled data are not new. Little has however been published on reconstruction strategies which not only yield a satisfactory dynamic 


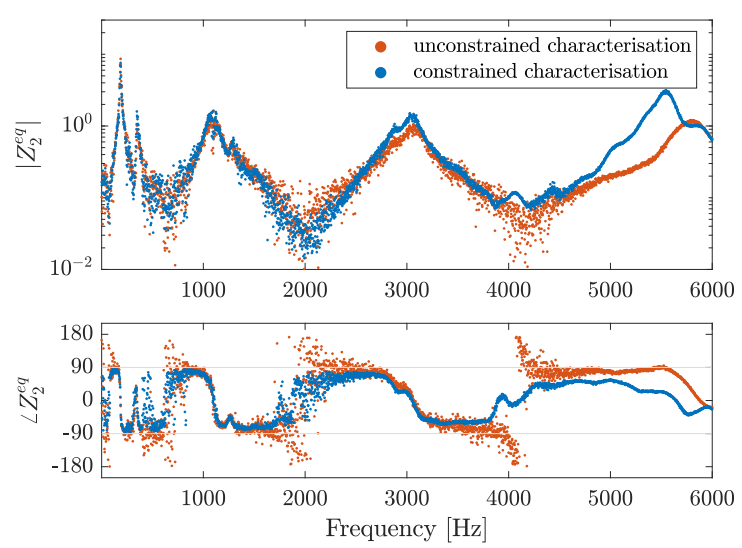

(a) Noisy input impedance $Z_{2}^{e q}(2,2)$
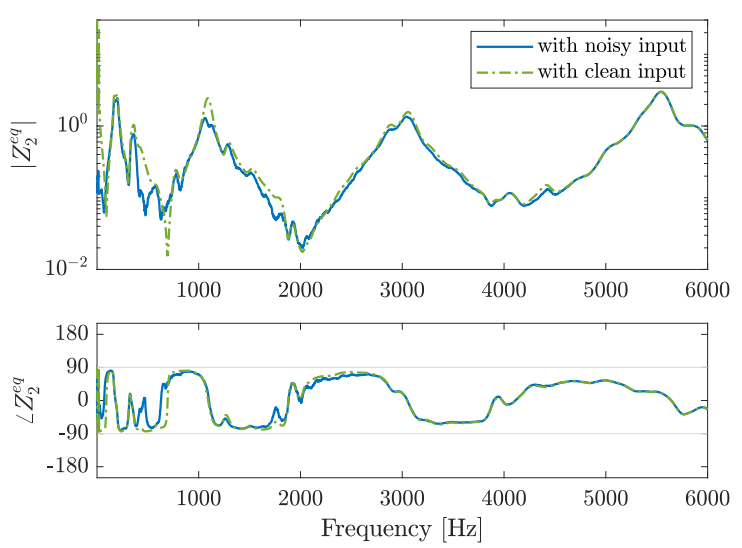

(b) Averaged input impedance $Z_{2}^{e q}(2,2)$

Figure 12: Input impedance for the 2nd DoF when noisy data are given as input to the system characterisation routine. In Figure 12a the impedance obtained with an unconstrained inverse characterisation (red dots) is compared with the one evaluated with the proposed methodology (blues dots). In Figure $12 \mathrm{~b}$ the moving average of the latter (blue solid line) is compared with the moving average of the physical impedance evaluated without any input noise (green dashed line). (For interpretation of the references to colour in this figure, the reader is referred to the web version of this article.)

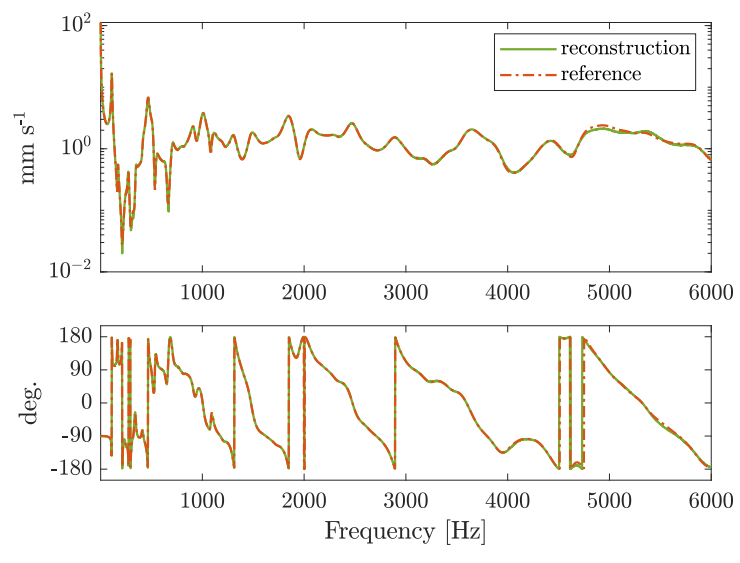

(a) out-of-plane velocity at $R_{1}$
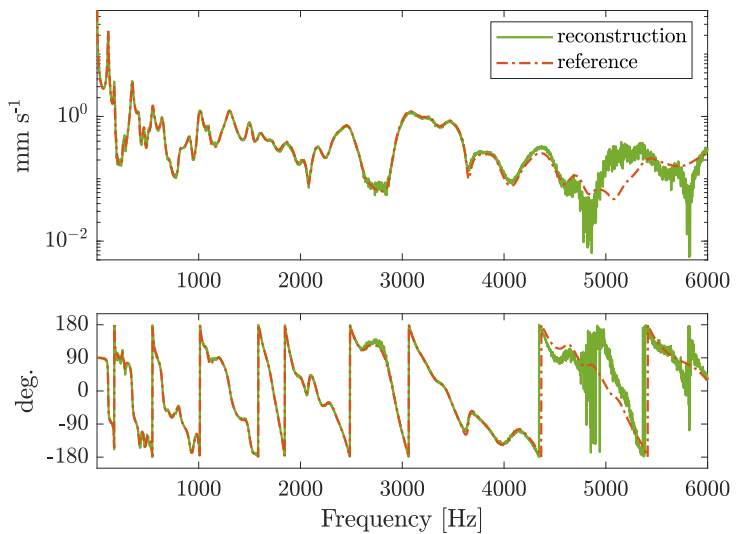

(b) out-of-plane velocity at $R_{2}$

Figure 13: Out-of-plane velocity of a point located on the source $13 \mathrm{a}$ and receiver $13 \mathrm{~b}$ subsystem. The reconstruction is evaluated with the substructuring approach when noisy FRFs are given as input and it is compared with the reference, evaluated using the fully coupled system.

system response but also assure the physical consistency, more in particular the reciprocity and passivity, of the resulting subsystem. In this paper a system identification procedure which assures such physical consistency is presented. Without limiting its generality, this method is here applied for the characterisation of subsystems connected along a line. The use of a small set of coupling points to approximate the dynamic behaviour of the continuous interface leads to a violation of fundamental properties of linear structural dynamic systems. The physically consistent characterisation consists then of three steps. Firstly, a reduced impedance matrix of the receiver subsystem is evaluated by solving a least-square problem. Constraints of symmetry and real semipositiveness of the diagonal entries are here introduced. Since they represent necessary but not sufficient conditions for passivity, the small negative eigenvalues of the impedance matrix's 
real part are filtered out in a second step. This ensures positive realness of the impedance matrix and, therefore, passivity. A limiting frequency $f_{\text {lim }}$ was introduced above which the negative eigenvalues increased steeply indicating the high frequency limit of the procedure. Finally, a second least-square problem is carried out in order to obtain a solution which, besides being physically consistent, represents the best fit of the coupled system data taken as input. Numerical experiments on a coupled beam-plate system showed accurate reconstructions with physically consistent subsystems and suggested that $f_{\text {lim }}$ corresponds with the Nyquist frequency. The scope of the current paper is purely methodological. A relatively simple test case has deliberately been chosen in order to create a controllable environment in which the results of the new methodology could be studied avoiding pollution by numerical and modelling errors and with the possibility to carry out well controlled noise sensitivity studies. A first test with noisy input data suggests that the methodology is robust also in presence of data pollution. Future research could address large numerical models having complex geometries, surface contacts and experimentally characterized subsystems.

\section{Acknowledgements}

The research work of Nicola Contartese has been funded by the European Commission within the ETN Marie Skłodowska Curie project PBNv2 (GA 721615). The authors acknowledge the financial support of the COMET K2 - Competence Centers for Excellent Technologies Programme of the Federal Ministry for Transport, Innovation and Technology (BMVIT), the Federal Ministry for Digital, Business and Enterprise (BMDW), the Austrian Research Promotion Agency (FFG), the Province of Styria and the Styrian Business Promotion Agency (SFG).

\section{References}

[1] W. C. Hurty, Dynamic analysis of structural systems using component modes, AIAA journal 3 (4) (1965) $678-685$.

[2] R. Craig, M. Bampton, Coupling of substructures for dynamic analyses, AIAA Journal 6 (7) (1968) 1313-1319.

[3] R. H. MacNeal, A hybrid method of component mode synthesis, Computers \& Structures 1 (4) (1971) 581-601.

[4] S. Rubin, Improved component-mode representation for structural dynamic analysis, AIAA journal 13 (8) (1975) $995-1006$.

[5] B. Jetmundsen, R. L. Bielawa, W. G. Flannelly, Generalized frequency domain substructure synthesis, Journal of the American Helicopter Society 33 (1) (1988) 55-64.

[6] T. Lim, J. Li, A theoretical and computational study of the FRF-based substructuring technique applying enhanced least square and TSVD approaches, Journal of Sound and Vibration 231 (4) (2000) 1135-1157. doi:10.1006/jsvi.1999.2724

[7] T. G. Carne, C. R. Dohrmann, Improving experimental frequency response function matrices for admittance modeling, in: Proceedings of the Nineteenth International Modal Analysis Conference, 2006, pp. 143-156.

[8] P. Peeters, S. Manzato, T. Tamarozzi, W. Desmet, Reducing the impact of measurement errors in FRF-based substructure decoupling using a modal model, Mechanical Systems and Signal Processing 99 (2018) 384-402. doi:10.1016/j.ymssp. 2017.06 .020

[9] D. D. Klerk, D. J. Rixen, S. N. Voormeeren, General Framework for Dynamic Substructuring: History, Review and Classification of Techniques, AIAA Journal 46 (5) (2008) 1169-1181. doi:10.2514/1.33274

[10] J. O'Callahan, P. Avitabile, R. Riemer, System equivalent reduction expansion process, in: Proc. of the 7th Inter. Modal Analysis Conf., 1989, 1989, pp. 29-37.

[11] H. Bonhoff, B. Petersson, The influence of cross-order terms in interface mobilities for structure-borne sound source characterization: plate-like structures, Journal of Sound and Vibration 311 (1-2) (2008) 473-484.

[12] S. Donders, B. Pluymers, P. Ragnarsson, R. Hadjit, W. Desmet, The wave-based substructuring approach for the efficient description of interface dynamics in substructuring, Journal of Sound and Vibration 329 (8) (2010) $1062-1080$.

[13] V. Meyer, L. Maxit, J.-L. Guyader, T. Leissing, C. Audoly, A condensed transfer function method as a tool for solving vibroacoustic problems, Proceedings of the Institution of Mechanical Engineers, Part C: Journal of Mechanical Engineering Science 230 (6) (2016) 928-938. doi:10.1177/0954406215615627

[14] P. Ind, D. Ewins, Impedance based decoupling and its application to indirect modal testing and component measurement: a numerical investigation, in: Proceedings of the Twenty First International Modal Analysis Conference, Kissimmee, FL, 2003.

[15] W. D'Ambrogio, A. Fregolent, Promises and pitfalls of decoupling procedures, Proceeding of 26th IMAC. Orlando (USA) (2008).

[16] S. Voormeeren, D. Rixen, A family of substructure decoupling techniques based on a dual assembly approach, Mechanical Systems and Signal Processing 27 (2012) 379-396.

[17] W. D'Ambrogio, A. Fregolent, Decoupling of a substructure from modal data of the complete structure, in: Proceedings of ISMA, vol. 2004, 2004, pp. 2693-2706.

[18] W. D'Ambrogio, A. Fregolent, Prediction of substructure properties using decoupling procedures, in: Proceedings of EURODYN, 2005. 
[19] W. D'Ambrogio, A. Fregolent, Inverse dynamic substructuring using the direct hybrid assembly in the frequency domain, Mechanical Systems and Signal Processing 45 (2) (2014) 360-377.

[20] A. Moorhouse, T. Evans, A. Elliott, Some relationships for coupled structures and their application to measurement of structural dynamic properties in situ, Mechanical Systems and Signal Processing 25 (5) (2011) 1574-1584. doi: 10.1016/j.ymssp.2010.12.016

[21] C. Höller, B. M. Gibbs, Indirect determination of the mobility of structure-borne sound sources, Journal of Sound and Vibration 344 (2015) 38-58. doi:10.1016/j.jsv.2015.01.011

[22] J. Zhen, T. C. Lim, G. Lu, Determination of system vibratory response characteristics applying a spectral-based inverse sub-structuring approach. Part I: analytical formulation, International Journal of Vehicle Noise and Vibration 1 (1-2) (2004) 1-30.

[23] Z.-W. Wang, J. Wang, Inverse substructure method of three-substructures coupled system and its application in producttransport-system, Journal of Vibration and Control 17 (6) (2011) 943-951.

[24] J. Wang, Z.-w. Wang, L.-x. Lu, Step-by-step decoupling method for inverse substructuring analysis of a three-component coupled packaging system, Journal of Vibration and Control 21 (4) (2015) 676-683.

[25] P. Wang, J. Wang, T. C. Lim, L. Lu, L. Pan, A strategy for decoupling of nonlinear systems using the inverse substructuring method and the parametric modal identification technique, Mechanical Systems and Signal Processing 140 (2020) 106695.

[26] P. Sjövall, T. Abrahamsson, Substructure system identification from coupled system test data, Mechanical Systems and Signal Processing 22 (1) (2008) 15-33. doi:10.1016/j.ymssp.2007.06.003.

[27] M. Grialou, Vibro-acoustics substructuring: Combining simulations and experimental identification of subdomains for low frequency vehicle acoustics, Ph.D. thesis, INSA Lyon (2018).

[28] S. Rubin, Transmission matrices for vibration and their relation to admittance and impedance, Journal of Engineering for Industry 86 (1) (1964) 9-21. doi:10.1115/1.3670463.

[29] M. S. Allen, D. Rixen, M. Van der Seijs, P. Tiso, T. Abrahamsson, R. L. Mayes, Substructuring in Engineering Dynamics, Springer, 2020.

[30] Y. I. Bobrovnitskii, A theorem on the representation of the field of forced vibrations of a composite elastic system, Acoustical Physics 47 (5) (2001) 507-510. doi:10.1134/1.1403536

[31] J. Meggitt, A. Moorhouse, On the completeness of interface descriptions and the consistency of blocked forces obtained in situ, Mechanical Systems and Signal Processing 145 (2020) 106850.

[32] K. J. Åström, B. Wittenmark, Adaptive Control, Addison Wesley, 1989.

[33] J. C. Willems, Realization of systems with internal passivity and symmetry constraints, Journal of the Franklin Institute 301 (6) (1976) 605-621.

[34] P. Triverio, S. Grivet-Talocia, M. S. Nakhla, F. G. Canavero, R. Achar, Stability, causality, and passivity in electrical interconnect models, IEEE Transactions on Advanced Packaging 30 (4) (2007) 795-808.

[35] D. S. Watkins, Fundamentals of Matrix Computations, Vol. 64, John Wiley \& Sons, 2004.

[36] P. Sjövall, T. Abrahamsson, Component system identification and state-space model synthesis, Mechanical Systems and Signal Processing 21 (7) (2007) 2697-2714. doi:10.1016/j.ymssp.2007.03.002

[37] S. Boyd, L. Vandenberghe, Convex Optimization, Cambridge University Press, 2004.

[38] S. Diamond, S. Boyd, CVXPY: A Python-Embedded Modeling Language for Convex Optimization, Journal of Machine Learning Research 17 (83) (2016) 1-5.

[39] A. Domahidi, E. Chu, S. Boyd, ECOS: an SOCP solver for embedded systems, in: 2013 European Control Conference (ECC), IEEE, 2013, pp. 3071-3076.

[40] Siemens Product Lifecycle Management Software Inc., NX Nastran 12. Basic Dynamic Analysis User's Guide (2017).

[41] Python Software Foundation, The Python Language Reference (2018).

[42] J. Meggitt, A. Moorhouse, A. Elliott, On the problem of describing the coupling interface between sub-structures: an experimental test for 'completeness', in: Dynamics of Coupled Structures, Volume 4, Springer, 2018, pp. 171-182. 